\begin{tabular}{ccc}
\hline International Journal of Engineering \& Technology, $7(2.32)(2018) 177-180$ \\
SPC \\
Website: ww w. sciencepubco.com/index.php/IJET \\
Research paper
\end{tabular}

\title{
A Survey on Prevention of Overfitting in Convolution Neural Networks Using Machine Learning Techniques
}

\author{
Dr.M.R.NarasingaRao ${ }^{1}$, V Venkatesh Prasad ${ }^{2}$, P.Sai Teja ${ }^{2}$, Md.Zindavali ${ }^{2}$, O.Phanindra Reddy ${ }^{2}$ \\ ${ }^{I}$ Professor, Department of CSE, KL E F, Guntur. \\ ${ }^{2}$ Department of CSE, KLE F, Guntur. \\ *Corresponding author E-mail: ramanarasingarao@kluniversity.in:
}

\begin{abstract}
Deep neural nets with a vast quantity of parameters are very effective machine getting to know structures. However, overfitting is an extreme problem in such networks. Massive networks are also sluggish to use, making it difficult to cope with overfitting by combining the predictions of many distinct large neural nets at test time. Dropout is a method for addressing this problem. The important thing concept is to randomly drop units (at the side of their connections) from the neural network for the duration of education. This prevents units from co-adapting an excessive amount of. during schooling, dropout samples from an exponential quantity of various "thinned" networks. At take a look at the time, it is simple to precise the impact of averaging the predictions of plenty of these thinned networks through in reality using a single unthinned network that has smaller weights. This considerably minimize overfitting and provides fundamental enhancements over other regularization techniques. We show that dropout enhance the overall performance of neural networks on manage gaining knowledge of obligations in imaginative and prescient, speech reputation, document type and computational biology, acquiring today's effects on many benchmark facts sets.
\end{abstract}

Keywords: Machine Learning, Connvultion Neural Network, Overfitting,Dropout

\section{Introduction}

A previous couple of years, Machine learning getting to know has brought about very good performance on a ramification of troubles, including visible reputation, speech recognition and Image processing. amongst specific varieties of neural networks. $\mathrm{CNN}$ had been maximum substantially studied. Presently on the fast boom in the quantity of the annotated facts and the extremely good enhancements inside the strengths of pix processor gadgets, the research on convolutional neural networks has been emerging swiftly and done modern effects on various duties. in this paper, we provide a wide survey of the recent advances in convolutional neural networks. we utilize the upgrades of CNN on special aspects, inclusive of layer layout, activation function, loss characteristic, regularization, optimization and fast computation. besides, we additionally introduce various packages of convolutional neural networks in laptop imaginative and prescient, speech and natural language processing.

\section{Prediction of Neural Networks}

An approach to computing and to information of the human brain. This technique isn't something new. The primary artificial neuron become produced in 1943 with the aid of the neurophysiologist Warren McCulloch and the truth seeker water pits. in the course of the 1960s, for reasons which are out of the scope of this article, humans turned away from neural networks and concentrated inside the symbolic aspect of synthetic intelligence. handiest inside the 1980s scientists saw the real capability of neural networks Neural networks take an extraordinary approach to hassle fixing than that of conventional computers. Traditional computers use an algorithmic technique i.e. the pc follows a fixed of commands so that it will clear up a trouble. Until the precise steps that the pc needs to comply with are acknowledged the laptop can't remedy the hassle. That restricts the problem-solving functionality of conventional computers to troubles that we already apprehend and understand the way to clear up. But computer systems might be a lot greater benefit if they could do things that we don't precisely recognize how to do.

\section{Adaptive Features Of CNN \\ Capability to derive meaning from complicated Accuracy infor- mation}

Extract styles and locate tendencies which are too complex to be observed by either human beings or other Machine techniques

Adaptive learning

Real-Time Operation

\section{Artificial Neural Networks}

These NN are a part of "Artificial intelligence". The artificial neural network is a device easygoing model based totally on the human mind or brain. the path is going through many names, which includes connectionism, parallel allotted processing, neurocomputing, herbal sensible structures, system gaining knowledge of algorithms, and Artificial neural networks

Artificial neural networks are preferred mathematical modules similar to our anxious machine in a mathematical version of a neuron, neuron transmitters effect an association of weights which 
makes an impact on associated facts signals the neuron drive is then processed as the weighted entirety of the statistical indicators, being modified via the exchange paintings. the specification is achieved by using altering of weights as per picked getting to know calculation.

1. Prediction: prediction of output variables with the help of entering values.

2. Classification: It tactics the classification degree the use of entering values.

3. Affiliation of facts: It plays each category and identity of mistakes.

4. Conceptualization of records: estimation of inputs which proceed in group family members

A multi-layered neural gadget and a faux neuron are proven in beneath the determine. each neuron is described by a motion level, a yield esteem, a wattle of facts institutions, an inclination esteem, lastly a wattle of yield institutions. the one's segments of the unit are portrayed numerically through proper esteems. in this way, every undertone possesses a synaptic weight that characterizes the effect of the unescapable contribution on the unit impulse restriction.

\section{Literature Survey}

In the 1990s, we saw big advancements in each chess and in stock buying and selling.

one of the reasons that the chess changed into one of the first a success device mastering programs become the availability of big quantities of labeled data. every most important chess recreation (and plenty of small ones) inside the ultimate a hundred and fifty years has been meticulously recorded and studied. and these kinds of facts use the name widespread notation for pieces, actions, the board, and so forth.

So there may be facts from thousands and thousands of games easily available - all inside the equal format. and there is isn't a lot of facts - the common wide variety of actions in the chessgames database is just forty not most effective is the chess information available, labeled, and comparatively small ... it was additionally really clean to get (you don't want to make a big $\mathrm{BD}$ deal). and the records on chess games did no longer have a whole lot of privateness implications to the players ... so it may be without problems allotted without fear it might be used for nefarious functions.

\section{Machine Learning Techniques}

Machine Learning was an exploit to link and execute in the computer that performed and run through the program that runs inbuilt In the past few years, machine learning was performed many wonders such as self-learning of driving cars, speech recognition, construction of web-search, and a vast improvement in understanding the humans Machine learning was un-doubtfully became very important that performs every minute operation in our dayto-day daily life. Numerous specialists, in addition, think it is the weightier tideway to proceeds ground towards human-level AI., you will deal with the most helpful machine learning strategies and continues work on executing them and inspiring them to work for yourself. All the increasingly significantly, we will retrospect the output which is expected to rapidly and intensely use these procedures to new issues.

This implicit quality which presents origin to machine learning, data mining, and statistical pattern recognition,it undergoes different strategies .

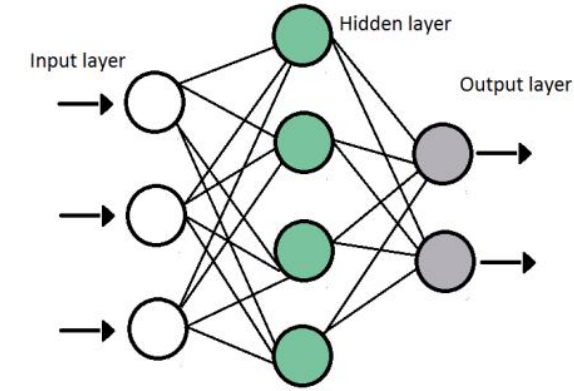

Artificial Neuron Representation

The basic computational element (model neuron) is frequently called a node or unit. it gets input from a few other devices, or perhaps from an external supply. each input has a related weight $\mathrm{w}$, which may be modified if you want to version synaptic mastering. the unit computes a few feature $f$ of the weighted sum of its inputs

- weighted sum is Known as the net input to unit $\mathrm{i}$,.

- wij represents to the weight from unit $\mathrm{j}$ to unit $\mathrm{i}$ (not the other way around).

- wij represents to the weight from unit $\mathrm{j}$ to unit $\mathrm{i}$.

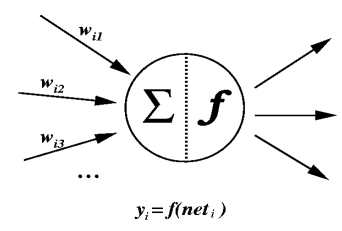

$y_{i}=f\left(\sum_{j} w_{i j} y_{j}\right)$

\section{Applications}

Neural Network packages can be grouped into following classes:

\section{Clustering:}

A clustering set of rules explores the similarity between patterns and places similar patterns in a cluster Good recognized packages include data mining and data compression

\section{Classification/Pattern recognition:}

The assignment of sample popularity is to assign an entered pattern (like a handwritten image) to one in every of many lessons. This category includes algorithmic implementations which include associative memory.

\section{Convolution Neural Networks}

The machine learning method which has been developed after the brain neuron structure which helps in Identification of different types of parameters and this consists of a network of intellect units know as neurons. These neurons prepare how to analyse given signals (e.g. image of a Dog) into identical output signals (e.g. the label "Dog"), creating the foundation of automated recognition

For example of automatic image recognition. The process of verifies whether a picture consists of a dog having an activation function. If the picture mimics prior dog photos that the neurons in neural network have seen before (seeing for a while in loop), the tag "Dog" would be activated. Hence, the more tagged in images the neurons are exposed to, it learns and analyses how to recognize other untagged images. This process is called training neurons. 


\section{Classification In CNN}

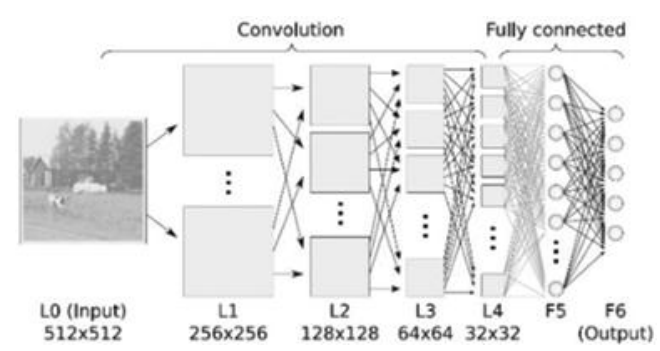

\section{Convolution}

convolution filters receive input signal these are actually the first layers. Convolution is a method in which the programmers attempts to label the given input signal by what it has already analyzed within the parameters. if the given input signal is preceding to ba a dog ,pictures it has seen before in neural network data hub, then "Dog" reference sign can be colaborated into, or convolved with, the given data. The ensuing output signal is then handed directly to the following layer.

Convolution have a good property of translational invariant. It says that every convolution filter represents a feature of interest (E.g whiskers, fur), and the CNN algorithm analyses which features comprise the output and then reference is set to be a defined object ('dog' here).

\section{Subsampling}

Different inputs from the convolution layer can be easily lessen the responsive of various filters to versions and noise. The processes is called smoothing these methods to reduce and separate is called subsampling, and can be carried out by way of taking mean or taking the most over a pattern of the sign. For photograph alerts of subsampling include reducing the dimensions of the picture, or decrementing the coloration contrast throughout purple, inexperienced, blue (rgb) channels

\section{Activation}

The activation layer controls signal to a directional flow from one layer to the subsequent, this processes is similar to how neurons are fired in our brain. The end product alerts which can bond perfectly related to previous references which would possibly spark off more neurons, allowing signals to be migrate extra efficiently for better identification.

CNN is accustomed with an intensive type of complicated activation capabilities to version signal propagation, the maximum commonplace feature being The Rectified Linear Unit (relu), that's desired for its premium education velocity.

\section{Fully Connected}

The very Final layers of the community are fully associated, which means that neurons of prior layers are linked to all neuron of the network in consecutive layers. this mimics immoderate degree reasoning wherein all adequate paths from the starting to resultant are Taken.

\section{Loss}

The training of the neural network is important due learning of patterns , there's additional layer known as the loss layer. This deposit gives feedback to the neural network on whether it diagnosed inputs effectively, and if no longer, how some distance off its makes an assumption in a probability chart. This enables to manual the neural community to enhance the proper ideas as it trains itself for future predictions. This is the last layer at some point of schooling.

\section{Pooling}

Convolutional networks is additionally included with neighbor- hood or universal pooling layers, which predicts the result of cluster containing neuron in every subsequent layer. Max pooling grabs fee from every of a neurons in cluster at the earlier layer. Every other example is mean pooling, which handles mean value from clustering methods of neurons on the previous layers.

\section{Weight}

CNN's shares different weights in every convolutional er ,having same filter are convoluted by each receptive field which reduces load on $\mathrm{db}$ or memory and magnifies its ability.

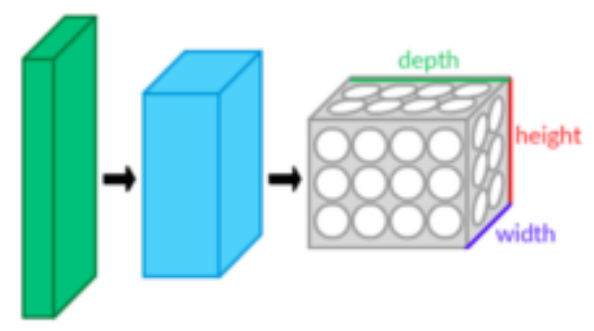

Convolutional neural networks are perceiving theory of the working of brain, design behavior of a cerebral cortex CNNS have the subsequent different abilities .

\section{Dimensional Volumes of Unit Neuron:}

Various kinds of layers in convolution neural networks has neurons organized in $3 \mathrm{D}:$ depth, height, and breath . Neurons interior layers are linked to handle a tiny place of them known as the receptive discipline. Different varieties of layer are regionally and absolutely linked collected to design a convolution neural networks architecture.

\section{Local connectivity}

The idea of accepting fields, Convolution neural networks take advantage of relatively close storage locations with the aid of finding a connection of vast pattern deduction among neurons of neighboring layers. This structure consequently guarantees the learned "filters" make this best reaction to a active space nearby enter sample. Collection of such many layers results in non-linear filters that come to be increasingly larger scale (i.e. conscious of a bigger area of pixel region)

\section{Shared weights:}

Convolution neural networks every filters are repeated along the whole training sets. The repetition gadgets share the identical parameters (like bias,vecto and weight) and form a characteristic map. Because of this all the ability of a complex neuron having different features to defy itself. Continuous Replicating of gadgets in this Way lets in for features to be identifies no matter their role inside the sight view, hence consisting of the assets of translation symmetry .

\section{Convolutional layer}

The convolutional layer is the major part in Convultion Neural Network, The layer's boundaries include a set of capable of being learned different types of filters (or kernels), that have a small receptive discipline, however, increase thru the overall intensity of the entered quantity. Every filter is a complex neuron across the height and breath of the entered quantity, the dot product among the entries which are separated and ready for being tested which has a tendency to produce a 2D activation map of the filter. As the end product would be community learns filters that prompt while it vitalizes some unique kind of function at some spatial role within given input.

These filters are processed by a neural network which deals with high amount of relevant data which then is used to access inside the output extent can accordingly and analyze as a result of a 
neuron that appears in the tiny place within the input and stock indexes with neuron in the identical activation key.

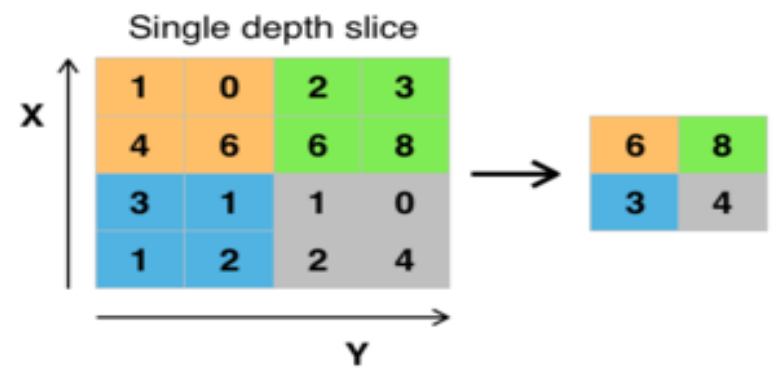

\section{Pooling layer}

Idea of CNNs within pooling, that's a shape of unstructured down-sampling. There are numerous unstructured functions to put in force to pooling is utmost unusual. It fix the entered image into a fixed of non-overlapping closed surfaced area a every sub-region, output to appropriate one. This instinct is the precise place of the character is much lower vital than its tough region relational to the other capabilities. The pooling layer depicts to regularly decrease the spacial length of the presenting a view to lessen the variety of specification and quantity of evaluation in the community and for this reason to additionally manipulate overfitting. It's far commonplace to time to time insert a pooling layer among consecutive convolution layers in a convolution neural network design. The pooling process affords any other shape of translation symmetry. The pooling layer operates on its own and on every intensity slice of the given input and resizes it spatially. the most commonplace form is a pooling layer with filters of size $2 \times 2$ implemented with a few decisive steps of 2 samples at each intensity slice within the input by way of 2 alongside each height and breath, leaving behind $75 \%$ of the activations. For example, each max survey is over 4 numbers. The depth measurement remains unharmed.

\section{Overfitting}

Overfitting happens when your model fits too well into the training set. It then becomes difficult for the model to generalize to new examples that were not in the training set. For example, your model recognizes specific images in your training set instead of general patterns. Your training accuracy will be higher than the accuracy of the validation/test set. So what can we do to reduce overfitting?

\section{Steps for reducing overfitting:}

1) Add more data

2) Use data augmentation

3) Use architectures that generalize well

4) Add regularization (mostly dropout, L1/L2 regularization are also possible)

5) Reduce architecture complexity.

\section{Dropout}

Dropout is a way that avert overfitting and gives a way of approximately combining proportionally Number of different neural network architect perfectly by temporary removing the units or nodes from the give train set. The probability fixed ' $p$ ' impartial of different units, $p$ may be selected the use of a validation set in between 0.5 and 1

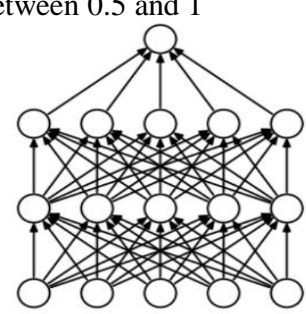

(a) Standard Neural Net

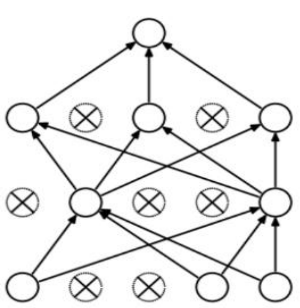

(b) After applying dropout.

\section{Conclusion}

In this Survey we have tried to Understand the Neural networks in image recognition, Representing of artificial neuron network classification in neural and also techniques to overcome overfitting ,Dropout is a technique for Developing neural networks by decreasing overfitting this approach turned into determined to enhance the performance of neural nets in a wide sort of utility domain names which includes image recognition Speech recognition biologicaldata.

\section{References}

[1]. Itamar Arel, Derek C Rose, and Thomas P Karnowski. Deep machine learning-a new frontier in artificial intelligence research [research frontier]. Computational Intelligence Magazine, IEEE, 5(4):13-18, 2010

[2]. Herbert Bay, Tinne Tuytelaars, and Luc Van Gool. Surf: Speeded up robust features. In Computer Vision-ECCV 2006, pages 404 417. Springer, 2006.

[3]. Yoshua Bengio. Learning deep architectures for ai. Foundations and Trends R in Machine Learning, 2(1):1-127, 2009.

[4]. Yoshua Bengio, Pascal Lamblin, Dan Popovici, and Hugo Larochelle. Greedy layerwise training of deep networks. Advances in neural information processing systems, 19:153, 2007.

[5]. Y-Lan Boureau, Jean Ponce, and Yann LeCun. A theoretical analysis of feature pooling in visual recognition. In Proceedings of the 27th International Conference on Machine Learning (ICML-10), pages $111-118,2010$

[6]. . Dan Ciresan, Ueli Meier, Jonathan Masci, and J"urgen Schmidhuber. A committee of neural networks for traffic sign classification. In Neural Networks (IJCNN), The 2011 International Joint Conference on, pages 1918-1921. IEEE, 2011.

[7]. . Dan Ciresan, Ueli Meier, Jonathan Masci, and J“urgen Schmidhuber. A committee of neural networks for traffic sign classification. In Neural Networks (IJCNN), The 2011 International Joint Conference on, pages 1918-1921. IEEE, 2011.

[8]. . Dan Claudiu Ciresan, Ueli Meier, Luca Maria Gambardella, and Jurgen Schmidhuber. Deep, big, simple neural nets for handwritten digit recognition. Neural computation, 22(12):3207-3220, 2010

[9]. Navneet Dalal and Bill Triggs. Histograms of oriented gradients for human detection. In Computer Vision and Pattern Recognition, 2005. CVPR 2005. IEEE Computer Society Conference on, volume 1, pages 886-893. IEEE, 2005.

[10]. ] S. Thrun and L. Pratt, Eds., Learning to learn. Norwell, MA, USA: Kluwer, 1998.

[11]. G. Webb, "Multiboosting: A technique for combining boosting and wagging," Machine learning, vol. 40, no. 2, pp. 159-196, 2000.

[12]. W. J. Dixon and A. M. Mood, "The statistical sign test," Journal of the American Statistical Association, vol. 41, no. 236, pp. 557-566, 1946

[13]. D. Saari and V. Merlin, "The Copeland method," Economic Theory, vol. 8 , no. 1 , pp. 51-76, 1996

[14]. ] J. N. Hooker, "Testing heuristics: We have it all wrong," Journal of Heuristics, vol. 1, pp. 33-42, 1995

[15]. ] T. Lane and W. Smart, "Why (PO)MDPs lose for spatial tasks and what to do about it," in Proceedings of the ICML 2005 Workshop on Rich Representations for Reinforcement Learning, 2005

[16]. M. Snel and S. Whiteson, "Multi-task evolutionary shaping without prespecified representations," in GECCO 2010: Proceedings of the Genetic and Evolutionary Computation Conference, July 2010, pp. 1031-1038.

[17]. Nitish Srivastava,, Geoffrey Hinton, Alex Krizhevsky, Ilya Sutskever, Ruslan Salakhutdinov Dropout: A Simple Way to Prevent Neural Networks from Overfitting 